ESJ Social Sciences

\title{
Savoir Traditionnel et Conservation de la Biodiversité dans le Rift Albertin: cas des Peuples Pygmée et Lega Riverains de la Réserve Naturelle d'Itombwe à l'Est de la RD Congo
}

\author{
Mangambu Mokoso Jean De Dieu
}

Professeur, Laboratoire de Systématique Végétale, Biodiversité \& Management des Écosystèmes (LSVBME), Département de Biologie, Faculté des Sciences, Université Officielle de Bukavu, RDC

\section{Aruna Sefu Josué}

Assistant, Institut Supérieur de Développement Rural de Kindu et Directeur Exécutif de 1'ONG Congo Basin Conservation Society CBCS-Network

\section{Byalungwe Muhindo Adrien}

Assistant, Faculté de Management et Sciences Économiques, Université du CEPROMAD-Bukavu, RDC

\section{Lubula Bawela Clovis}

Superviseur-Chef de Service de l'Environnement et Développement Durable, Expert en Communication Environnementale, Bukavu-Sud-Kivu, RDC

\section{Lwimo Mukenge Manassé}

Chef de Travaux, Université Libre des Pays des Grands Lacs - Bukavu,

Faculté de Santé et Développement, Département de Santé et

Environnement, Bukavu, RDC

\section{Mushangalusa Kidumbi Jackson}

Chef de Travaux, Faculté de Management et Sciences Économiques,

Université du CEPROMAD-Bukavu, RDC

\section{Kambale Kavusa Gentil}

Chercheur en Politique de Conservation et Management des Ecosystèmes forestiers, Projet World Sida, World Wild Fund of Nature, WWF- Sud Kivu, RDC

\section{Doi:10.19044/esj.2021.v17n32p319}

Submitted: 14 June 2021

Accepted: 09 September 2021

Published: 30 September 2021
Copyright 2021 Author(s)

Under Creative Commons BY-NC-ND

4.0 OPEN ACCESS

Cite As:

Jean De Dieu M.M., Josué A.S., Adrien B.M., Clovis L.B., Manassé L.M., Jackson M.K. \& Gentil K.K. (2021). Savoir Traditionnel et Conservation de la Biodiversité dans le Rift Albertin: cas des Peuples Pygmée et Lega Riverains de la Réserve Naturelle d'Itombwe à l'Est de la RD Congo. European Scientific Journal, ESJ, 17 (32), 319.

https://doi.org/10.19044/esj.2021.v17n32p319 


\section{Résumé}

Cet article se focalise sur la nécessité d'intégrer, de réconcilier les pratiques coutumières des peuples riverains (Pygmées et Lega) avec les pratiques de gestion moderne de conservation de la biodiversité afin de préserver et de protéger la Réserve Naturelle d'Itombwe (RNI, réserve de catégorie VI de l'UICN). Pour récolter les données sur le terrain dans les villages Lega et Pygmées proches de la RNI, trois techniques ont été utilisées (documentaire, focus group et entretiens semi-structurés). Les résultats obtenus nous ont permis de découvrir les modes de protection de la nature et les normes locales propices au développement durable dans la gestion et la conservation de la nature. Ils montrent qu'il est utile de prendre en compte les pratiques traditionnelles et les expériences vécues pour comprendre l'importance du monde vivant de ces deux peuples. Certaines plantes et certains animaux protégés par les Us et coutumes de deux peuples sont aussi des espèces protégées et interdites par la Convention sur le Commerce International des Espèces de Faune et de Flore Sauvages Menacées d'Extinction (CITES). Malheureusement, la forte croissance démographique, le christianisme, la modernité ainsi que la relation entre la pauvreté et la culture traditionnelle ont remis en cause ces pratiques culturelles et le nonrespect de coutume. Ces croyances, loin d'être stables et immobiles, sont souvent capables d'intégrer des formes de nouveauté et de s'adapter à des modalités variables. En dépit de ces obligations, les occasions s'offrent au niveau coutumier pour la bonne gestion de la nature. Il revient aux acteurs de la conservation tant nationale qu'internationale de s'approprier ces pratiques costumières pour une bonne gestion de la biodiversité.

Mots-clés: Us et coutumes, Gestion de la biodiversité, Réserve Naturelle d'Itombwe, RDC 


\title{
Traditional Knowledge and Biodiversity Conservation in the Albertine Rift: The Case of the Pygmy and Lega Peoples Living in the Itombwe Nature Reserve in Eastern DRC
}

\author{
Mangambu Mokoso Jean De Dieu \\ Professeur, Laboratoire de Systématique Végétale, Biodiversité \& \\ Management des Écosystèmes (LSVBME), Département de Biologie, \\ Faculté des Sciences, Université Officielle de Bukavu, RDC \\ Aruna Sefu Josué \\ Assistant, Institut Supérieur de Développement Rural de Kindu et Directeur \\ Exécutif de l'ONG Congo Basin Conservation Society CBCS-Network

\section{Byalungwe Muhindo Adrien} \\ Assistant, Faculté de Management et Sciences Économiques, Université du \\ CEPROMAD-Bukavu, RDC \\ Lubula Bawela Clovis
}

Superviseur-Chef de Service de l'Environnement et Développement Durable, Expert en Communication Environnementale, Bukavu-Sud-Kivu, RDC

\section{Lwimo Mukenge Manassé}

Chef de Travaux, Université Libre des Pays des Grands Lacs - Bukavu, Faculté de Santé et Développement, Département de Santé et

Environnement, Bukavu, RDC

Mushangalusa Kidumbi Jackson

Chef de Travaux, Faculté de Management et Sciences Économiques, Université du CEPROMAD-Bukavu, RDC

\section{Kambale Kavusa Gentil}

Chercheur en Politique de Conservation et Management des Ecosystèmes forestiers, Projet World Sida, World Wild Fund of Nature, WWF- Sud Kivu,

RDC

\section{Abstract}

This paper focuses on the need to integrate and reconcile the customs and practices of the riparian peoples (Pygmies and Lega) with the practice of modern management and conservation of biodiversity in order to preserve and protect the Itombwe Nature Reserve (INR, which is an IUCN Category VI Reserve). To collect field data in Lega and Pygmy villages bordering the INR, three techniques was used (documentary, focus group and individual interviews). The studies uncovered ways of protecting nature and local sacredness that are conducive to sustainable development in nature management and conservation. They show us that it is useful to take into account traditional practices and lived experiences to understand the 
importance of the living world of these two peoples. In addition, some plants and animals protected by local knowledge are among the species protected and prohibited by the Convention on International Trade in Endangered Species (CITES). The strong demographic growth, Christianity, modernity as well as the grafting between poverty and traditional culture have challenged these cultural practices and the respect of customs. These beliefs, far from being stable and immobile, are often able to integrate forms of novelty and adapt to changing modalities. In spite of these obligations, opportunities exist at the customary level the good management of nature. It is up to national and international conservation actors to appropriate these customary practices for good biodiversity management.

Keywords: Uses and customs, Biodiversity management, Itombwe Nature Reserve, DRC

\section{Introduction}

Pour mieux conserver la biodiversité et les aires protégées dans les pays du Sud, il est nécessaire d'impliquer les sociétés traditionnelles qui ont des relations avec leur environnement naturel depuis l'aube de l'humanité et maitriser des pratiques traditionnelles. D'après Roué, (2012), présentement la gestion des ressources naturelles basée sur les pratiques modernes ont montrés leurs limites (Pinton \& Grenand, 2007), contrairement aux stratégies traditionnelles dont la communication et la transmission de la connaissance étaient orales et ses connaissances acquises se sont transmises de générations en générations (Kohler, 2012; Mboa, 2015).

La prise en compte de ces Us et coutumes s'avère indispensable pour concilier les pratiques coutumières et culturelles avec les théories modernes de la conservation et gestion durable dans les aires protégées (Colchester, 2003 ; Sene et al., 2013 ; Mboa, 2015 ; Mangambu et al., 2012 ; Mangambu et $a l ., 2018)$. C'est pour cette raison, on pense que les savoirs traditionnels ou culturels se rapportent de la manière dont les individus comprennent le monde vivant et aussi leur intégration au sein des expériences vécues.

Le savoir n'est pas lié à une vérité objective ou inébranlable, mais s'il s'agit plutôt d'un système de compréhension culturelle subjective et conditionnée qui provient des mécanismes complexes et en constante évolution (Butare, 2003; Colchester, 2003; Houngnihin, 2005; Marlise et al., 2021). De cette manière, le savoir local implique la sélection, le rejet, la création, le développement et l'acquisition des informations. Ces processus sont liés aux contextes sociaux, environnementaux et institutionnels existant dans les sociétés traditionnelles (Coulibaly, 1995 ; Bisidi et al., 2008; Kyapa \& Maindo, 2017; Mangambu \& Kambala, 2019). 
Ces questions sur les savoirs traditionnels ne datent pas d'aujourd'hui. Ils ont toujours été au centre de nombreux débats aux dimensions scientifiques et locales variées et mettent en jeu des acteurs aux intérêts divers, que ce soit les chercheurs ou les porteurs de ces savoirs pour renforcer la conservation (Kingsbury, 1996; Maretti, 2003; Mboa, 2015).

Mboa (2015), Mangambu et al. (2015a), Kyale et Maindo (2017) considèrent que la persistance de ces connaissances naturalistes locales appartient à une tradition qui garantit une certaine ancienneté. Car, ce savoir traditionnel est une dynamique sur l'ensemble des systèmes de croyance et des pratiques qui comprend l'inventaire non écrit des ressources biologiques locales par les peuples indigènes selon leur tradition (Bouzou \& Yamba, 2008 ; Bikaba, 2013 ; Mubalama et al., 2018).

A l'Est de la République Démocratique du Congo (RDC), les communautés autochtones et voisines des aires protégées ont consacré certains paysages forestiers gratifiés de divers interdits pour faciliter l'organisation d'une diversité d'activités culturelles. Sous cette même perspective, certaines plantes et animaux ont été mises à l'abri de toute exploitation vue le rôle et la place qu'elles jouent dans le domaine du sacré, de la nourriture et/ou de la pharmacopée (Mangambu et al., 2015a ; Mangambu et al., 2015b). Malgré cette importance alimentaire, artistique, la médicinale,... force est de constater que de nos jours, ces ressources sont menacées par la croissance démographique, le surpâturage, la déforestation etc. (Maretti, 2003; Langewiesche, 2006 ; Agarwal, 2009; Mangambu et al., 2015b).

Les peuples riverains de la Réserve Naturelle d'Itombwe (RNI), à l'instar des autres communautés autour des aires protégées, développent dans leurs coutumes respectives, des pratiques liées à la conservation de la nature d'après leurs sagesses et éthiques environnementales expriment à travers des croyances religieuses. C'est une série de pratiques sacrées et culturelles (Mubalama et al., 2018).

C'est dans cette optique que nous avons mené cette recherche orientée sous forme des ateliers organisés avec les gardiens des coutumes et les communautés locales y compris les peuples pygmées des entités administratives abritant une partie de la RNI, notamment en chefferie des Basile, Wamuzimu et Lwindi dans le Territoire de Mwenga en Province du Sud Kivu.

L'objectif de cette recherche est d'identifier « les Us et coutumes » des populations Lega et Batwa (Pygmées) riveraines de la RNI dans ces trois Entités Territoriales Décentralisées (ETD). Il s'agit spécifiquement de savoir : $(i)$ les normes coutumières et les pratiques traditionnelles existantes ; (ii) le rôle des Us et coutumes dans la conservation des ressources naturelles et (iii) l'impact des changements socioculturelles et/ou sur leur pérennisation de ses savoirs traditionnels. 


\section{Cadre Methodologie}

\section{Réserve Naturelle d'Itombwe}

La Réserve Naturelle d'Itombwe (RNI), (Figure 1) est située à l'Est de la RDC dans la Province du Sud-Kivu, à l'Ouest du Lac Tanganyika, dans la chaîne montagneuse de Mitumba, sur le fossé du Rift Albertin (Doumenge \& Schilter 1997; Mubalama et al., 2018). Le sommet le plus élevé est le Mont Muhi (3 $475 \mathrm{~m})$. Ces massifs sont restés pendant très longtemps inaccessibles, très isolés, et donc très peu administrés, même pendant la période coloniale. Créée comme réserve en 1998, la RNI a bénéficié de l'Arrêté provincial $\mathrm{n}^{\circ} 01 / 008 / \mathrm{CAB} / \mathrm{GP}-\mathrm{SK}$ du $25 / 02 / 1998$ portant mesure de sauvegarde de la faune et de la flore des massifs d'Itombwe. C'est suite à l'Arrêté Ministériel $n^{\circ} 038 / \mathrm{CAB} / \mathrm{MIN} / \mathrm{ECN}-\mathrm{EF} / 2006$ du 11 octobre 2006 que la RNI a vu le jour. Le processus de délimitation participative qui constitue une étape clé pour le développement de la RNI, a débouché à la signature, par le Gouverneur de Province de l'arrêté ${ }^{\circ} 16 / 026 / \mathrm{GP} / \mathrm{SK}$ du 20 juin 2016 portant actualisation et configuration physique des limites de la RNI et cet après l'aval du Conseil Consultatif Provincial des Forêts (CCPF).

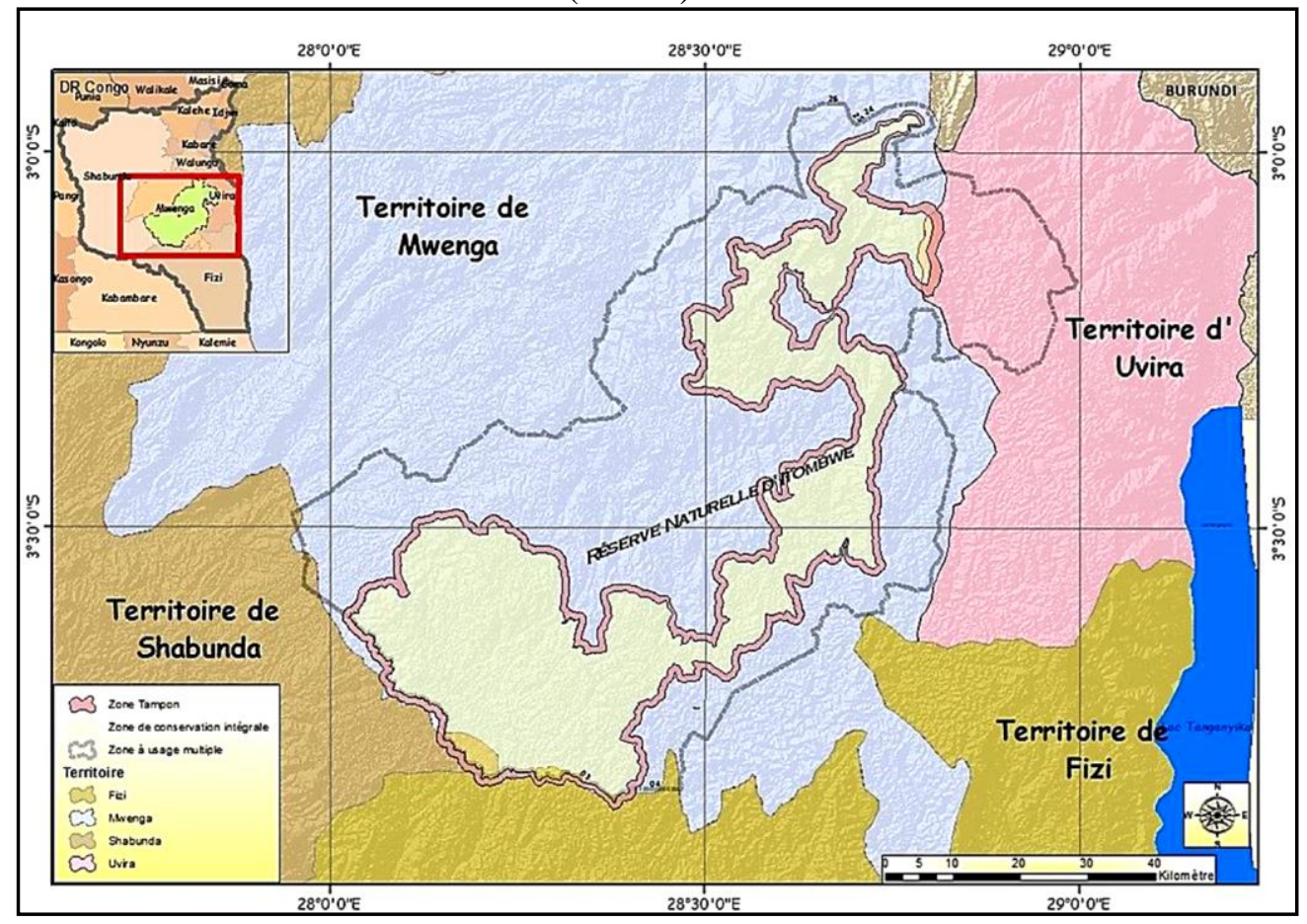

Figure 1. Localisation administrative de la RNI

(source : Plan d'Aménagement et de Gestion de la RNI, 2017).

A l'heure actuelle, ses limites sont connues, mais la démarcation des limites externes demeure une activité importante que l'Institut Congolais pour la Conservation de la Nature (ICCN) doit réaliser avec ses partenaires. La 
superficie de la RNI est de $5732 \mathrm{~km}^{2}$ répartie en trois zones à savoir : la zone de conservation intégrale $(36,3 \%)$, la zone tampon $(18,8 \%)$ et la zone à usage multiple (44, 9\%) (Plumptre et al., 2009).

D'après Doumenge et Schilter (1997), Plumptre et al. (2009), Greenbaum et Chifundera (2012) et Mangambu (2021); la flore des massifs d'Itombwe se trouve à la croisée de plusieurs grands ensembles phytogéographiques. A l'Ouest du massif, la flore appartient au «Centre d'endémisme guinéo-congolais » constitué des forêts ombrophiles planitiaires Guinéo-Congolaises.

Les formations végétales du Sud sont rattachées au «Centre régional d'endémisme zambézien » et les formations végétales d'altitude sont caractéristiques du « centre régional d'endémisme morcelé afro-montagnard ». Cet ensemble phytogéographique est constitué de forêts ombrophiles de transition, de forêts afromontagnardes indifférenciées et de végétation altimontaine. D'après Doumenge et Schilter (1997), Mangambu (2021); la RNI engorge un réseau hydrographique dense appartenant au bassin versant du fleuve Congo et du lac Tanganyika.

\section{Description De La Démarche Méthodologique \\ Techniques de collecte des données sur le terrain}

Pour récolter des données sur le terrain, nous avons mobilisé trois techniques. Il s'agit, entre autres documentaire, les focus group et des entretiens semi-structurés. Elles ont été utilisées afin de réunir les renseignements sur les valeurs et l'importance des Us et coutumes en matière de conservation. Les interdits coutumiers, leur mode de règlementation, les mythes et les rites ainsi que le rôle de la femme dans les sociétés traditionnelles ont été pris en compte par ces techniques de la récolte des données.

- Des documents d'archives ont été consultés aux chefferies (Basile, Lwindi et Wamuzimu), aux Divisions provinciales de l'Intérieur et de Culture, au Centre de Recherche des Sciences Naturelles (CRSN) de Lwiro et à l'INERA Mulungu.

- Les focus group ou carrefours ont été organisés auprès deux communautés riveraines de la RNI tendant cueillir les informations sur les pratiques ancestrales de la conservation de la nature. La pertinence des focus group dans cette étude se justifie par le fait que «les savoirs et pratiques locaux sont transmis oralement par les plus anciens aux jeunes et possèdent une forte dimension symbolique et sociale traditionnelle (Moreau et al. 2004; Touré, 2010; Gavard-Perret et al., 2011).

- Les interviews individuelles ont permis de recueillir les informations auprès des vieux et autres personnes-clés comme les gardiens de coutume au regard de la pertinence des renseignements culturelles qu'ils détiennent. 


\section{Identification des échantillons des plantes et les animaux}

Sur terrain, nous avons noté en langues locales les noms des espèces et des spécimens. Les traductions faites par les Botanistes et les Zoologistes appartenant chacun au peuple étudié, ont facilitées l'identification des quelques espèces des plantes et des animaux citées. Les spécimens non connues sur le terrain, ont été comparé aux spécimens des référence de musée et des herbiers du CRSN - Lwiro. Pour d'autres, on a fait référence aux travaux de Troupin (1979, 1982, 1985 et 1988), Bloesch et al. (2009), et Mangambu (2013). Les échantillons récoltés sur terrains se trouvent au CRSN - Lwiro pour une bonne conservation et servir aux études ultérieures.

\section{Analyse des données}

Nous avons effectué les analyses ci-après :

- Taux de réponse : Pour connaitre les divers usages des peuples étudiés, nous avons calculé le taux de réponse $(F)$ des espèces citées qui s'exprime par cette équation ci-dessous (Maregesi et al., 2007):

$$
F=\frac{s}{N} \times 100
$$

Où, $F$ : taux de réponse ; $S:$ nombre de personne ayant donné une réponse positive (Oui) pour l'utilisation de l'organe concerné ; $N$ : nombre total de personnes interviewées. Il indique les organes les plus utilisés pour chaque espèce dans le milieu et varie de 0 à 100.

- Niveau de connaissance des Us et coutumes : Le niveau de connaissance des Us et coutumes est calculé en évaluant la Fréquence de Citation (FC) qui est le nombre de fois que le répondant a mentionné une espèce citée par la population interviewée et ou pensant focus group. C'est un indice qui évalue la crédibilité des informations reçues sur le niveau de connaissance des aires protégées par les pratiques traditionnelles (Tardío \& Pardo-de-Santayana, 2008) :

$$
F C=\frac{\text { Nombre de citation }}{\text { Nombre total de citation }} \times 100
$$

- Tests d'analyse de variance (Kruskal-Wallis) : ont été utilisés pour comparer les différentes pratiques traditionnelles selon les tribus en utilisant le logiciel Minitab 14.0 (Tardío et Pardo-de-Santayana, 2008). Ensuite, nous avons comparé les traits d'usage entre les pygmées et les autres groupes ethnolinguistiques en utilisant le test $t$ de Student à partir du logiciel $\mathrm{R}$ version. 


\section{Identites, Tradition Et Mode De Gestion Traditionnelle Des Ressources Naturelles}

Peuple Batwa (Pygmee) des Chefferies de Basile, Lwindi et Wamuzimu

Les Pygmées sont, à l'origine, un peuple nomade vivant de la cueillette, de la chasse et de la pêche. Ils sont les premiers habitants des pays d'Afrique Centrale et en particulier les forêts de basse altitude et de montagnes dans la région de Grands Lacs. Ils furent rejoints, puis supplantés par les peuples sédentaires dont les bantous. Mais dans les chefferies étudiées, les Batwa sont des peuples migrants. Leur histoire les lie des leurs milieux d'initiation traditionnelle, ce qui leur donne une responsabilité dans la protection des ressources naturelles. Les «Batwa ou Mbuti» considèrent la terre, la forêt comme leur propriété privée, ils doivent les conserver pour des fins culturelles et traditionnelles.

Leurs connaissances coutumières servent de base à l'interrogation sur la conservation du milieu naturel. Ils fabriquent eux-mêmes leur médicament sur base des ossements des animaux sauvages et des plantes grâces aux savoirs reçus par les aïeux et transmis d'une génération à l'autre d'une manière perpétuelle.

\section{Spiritualité et cultures}

En ce qui concerne leur culture, ce peuple chante et danse dans un style assez distinctif. Pour leur croyance, il s'accorde à un rite monothéiste animiste. Il pratique le culte des aïeux et respectent «Zèngi ou Jengi», l'intermédiaire entre Dieu et les êtres humains, le protecteur des Batwa. Ils se consacrent à la croyance des esprits-animaux et des puissances de la nature. Les hommes «Mbuti», font aussi leur initiation appelée «lutende» (Figure 2), pendant des semaines d'isolement dans les forêts sacrées. 


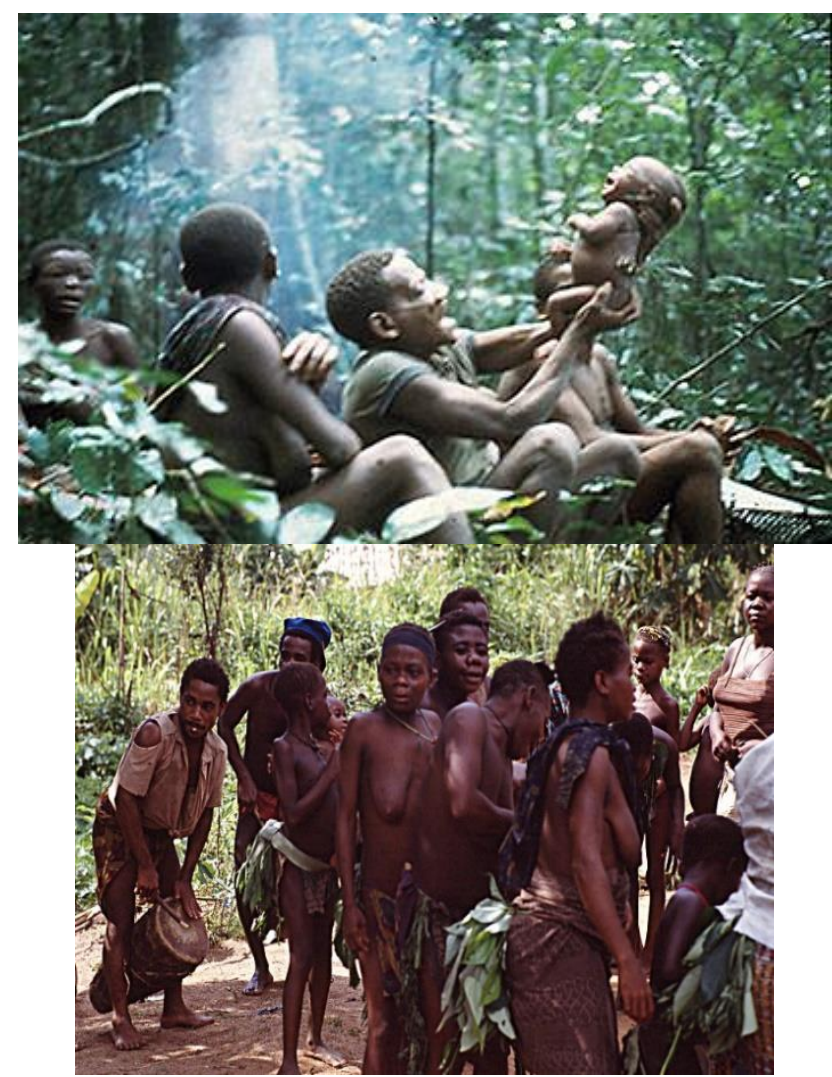

Figure 2. A gauche, initiation d'un enfant d'une année ; à droite, le "lutende", pendant des mois sacrés en forêt

Le lieu authentique et le contenu de ce rituel restent discrets, car c'est la clé de la transmission de leurs connaissances traditionnelles d'une génération à l'autre. Ils sont formés par les gardiens de coutume, ils maitrisent toutes les prohibitions, les sites et les cycles de chasse et de pêche. Par exemple, pendant la saison sèche, ils ne chassent pas, car les animaux vont mettre bas. Il y a aussi les animaux autorisés pour la chasse quel que soit la période d'interdiction, comme le «mokumbi » (le rat de Gambie), et ceux que l'on ne peut pas abattre, comme les grands singes. Ils ont leurs propres techniques classiques de conservation. Par exemple : les animaux que la loi moderne veut conserver sont déjà notre protection coutumière (Tableau 3 ). Ce sont les lois que aïeux ont léguées à la génération présente pour gérer la forêt et ses richesses.

\section{Genres}

En matière de travail dans la société des Batwa, on observe une répartition des tâches en fonction du sexe. La chasse est reconnue une tache très difficile (Figure 3 ) reste l'apanage de homme, la pêche et la cueillette 
relèvent plutôt des activités de la femme. Et cette dernière joue un rôle essentiel dans la communauté Batwa.

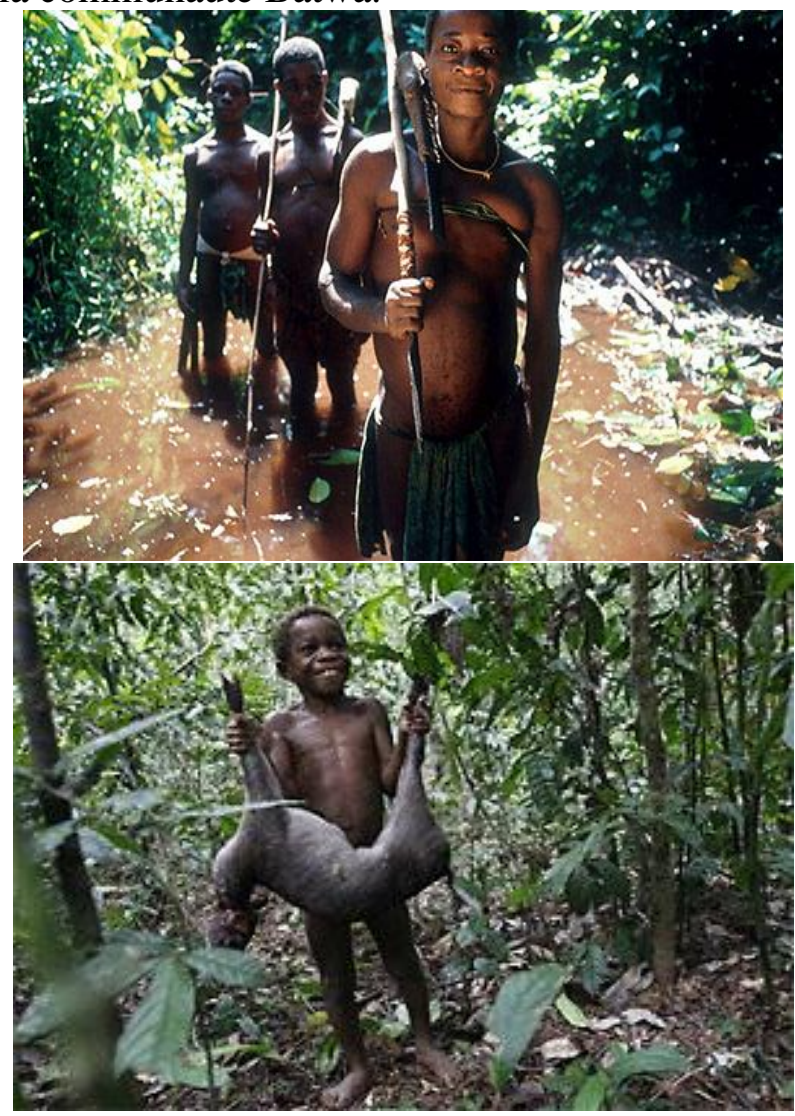

Figure 3. A gauche, les hommes Batwa partent à la chasse collective; à droit une antilope tombée dans un filet.

Elle est responsable de la gestion de l'épargne du ménage. Elle occupe des achats, des ventes, de la cuisine, des investissements, des travaux agricoles et domestiques, la transmission des messages et la sensibilisation des jeunes. Parfois, elle est estimée comme intercesseur entre l'homme et son «dieu de la chance »en pratiquant quelques rites pour apporter la chance à son époux est à la chasse. En général, dans la société traditionnelle Batwa ou Mbuti, les hommes chassent et collectent le miel, les femmes collectent les bois et toute la famille collecte les chenilles et à la chasse au filet (Figure 3). Il arrive très souvent qu'au sein d'une famille, chacun des membres, femmes comme hommes, associe à toutes les activités pour obtenir une bonne récolte ou un produit de chasse. Les Pygmées sont reconnus comme des grands connaisseurs de la vie de forêt et de l'utilité des plantes médicinales. 


\section{Connaissance traditionnelle des Batwa}

Les systèmes traditionnels de gestion de la biodiversité sur les Us et coutumes révèlent d'une importance particulière sur le caractère sacré, confidentiel et même secret d'une partie de ces connaissances chez ces peuples. Nos recherches nous ont amené aux réponses de ces connaissances dans les points ci-dessous :

- Les espèces végétales d'usages divers totalement protégées : Ce peuple est connecté quasiment à leur spiritualité dans les milieux sacrés en forêt. Suivant les réponses aux interviews, ils répondent ceci : "Nous savons comment protéger notre forêt car personne ne la maitrise mieux que nous. Nous savons la période à laquelle et dans quel endroit où les espèces animales mettent bas, où elles se reposent, et à quelle intervalle de l'année il ne faut jamais les tuer ». Dans cette forêt, nous trouvons le bois pour construire nos cases, les produits forestiers non ligneux (les fruits et les chenilles...) pour manger, soigner nos maux et les plantes d'agroforesterie ainsi que les espèces des plantes totalement protégées.

Dans le Tableau 1 ci-dessous, sont présentées les espèces qui sont totalement protégés.

Tableau 1. Les espèces végétales protégées par la coutume de «Batwa » ou pygmée

\begin{tabular}{ccl}
\hline Noms vernaculaires & Noms Scientifiques & Utilité \\
\hline Alya masilya & Microdesmis pierlotiana & Chenilles et médicinale et mystiques \\
\hline Am'muuta & Pseudoprosopsis claessensii & Chenilles et médicinale et mystiques \\
\hline Kasela & Uapaca benguelensis & chenilles \\
\hline Kasela-Kulongo & Uapaca paludosa & chenilles \\
\hline Kisekenumpa & Tetrapleura tetraptera & Chenilles et médicinale et mystiques \\
& & \\
\hline Kisela-sela & Uapaca corbisieri & Chenilles et culturelle \\
\hline Lukundu ou Luungu & Piptadeniastrum africanum & $\begin{array}{l}\text { Chenilles, agroforesterie, médicinale } \\
\text { mystiques }\end{array}$ \\
\hline Mseke-seke & Cathormion leptophyllum & $\begin{array}{l}\text { Chenilles, médicinale, agroforesterie } \\
\text { et mystiques }\end{array}$ \\
\hline Msela & Uapaca zanzibarica & chenille \\
\hline Mshishi & Cynometra hankei & Chenille et culturelle à l'intronisation \\
\hline Mshishi & Monopetalanthus microphyllus & Chenilles et agroforesterie \\
\hline Museka & Julbernardia seretii & Chenilles et agroforesterie \\
\hline Musela & Uapaca guineensis & chenille \\
\hline Moombi & Annonidium manni & Alimentaire \\
\hline Musuku & Canarium schweinfurthii & $\begin{array}{l}\text { Chenilles et l'accent pour la lumière } \\
\text { chasse les faux esprits }\end{array}$ \\
\hline Lufili & Scorodophloeus zenkeri & Chenille, Alimentaire et faux esprits \\
\hline Butunga-Mbala & Laea guineensis & Alimentaire \\
\hline
\end{tabular}

Au total, 17 espèces végétales ont été recensées comme espèces totalement protégées par les Batwa. 
Certaines espèces comme, Microdesmis pierlotiana, Scorodophloeus zenkeri Canarium schweinfurthii, Cathormion leptophyllum ont un taux de réponse élevé de valeur de l'intervalle de respectives [82-91] d'usage ethnobiologique par rapport aux autres compte tenu de leur valeur culturelle.

- Plantes d'usage rationnel : Les plantes sont l'élément principal des soins connues par les Pygmées. Ils utilisent des écorces, des racines, des feuilles et des épines etc. pour faire des décoctions, des tisanes, de la poudre pour scarification, des injections par voie nasale pour leurs différents soins. Dans le tableau 2 ci-dessous, nous complétons la liste des plantes médicinales tel que révélées par les pygmées environnant la RNI dans le territoire de Mwenga.

Tableau 2. Les espèces médicinales utilisées rationnellement par les Batwa

\begin{tabular}{cc}
\hline Noms vernaculaires & Noms Scientifiques \\
\hline Achukwe-Chukwe, Mushumba-Humba & Prunus africana \\
\hline Bushahi, Musebu & Lebrunia bushaie \\
\hline Kantonko & Rauvolfia congolana \\
\hline Katondo & Rauwolfia vomitoria \\
\hline Kilondolondo & Ficus elastica \\
\hline Kufa & Biophytum helenae \\
\hline Kyanina-Kya-Kabi & Struchnos memecyloides \\
\hline Kyanina-Kya-Kabi & Struchnos scheffleri \\
\hline Lungusu & Alchornea cordifolia \\
\hline Mulemansu-Ha Mabele & Kigelia africna \\
\hline Musela & Uapaca benguelensis \\
\hline Mutondo & Alstonia congensis \\
\hline Mutungulu & Aframomum laurentii \\
\hline Yamwasamusa & Anisoppapus africanus \\
\hline
\end{tabular}

Il ressort de ce tableau que le niveau de connaissance des Us et coutumes des espèces présentant une forte connaissance par les pygmées : ces espèces sont respectivement Prunus africana $(\mathrm{FC}=8,98)$, Spondianthus preussii $(\mathrm{FC}=8,16)$, Lebrunia bushaie $(\mathrm{FC}=7,9)$, Kigelia africna $(\mathrm{FC}=7,68)$. La connaissance des autres espèces leur connaissance est moins de 7,5. Le test d'ANOVA montre que les connaissances liées aux Us et coutumes des ressources ligneuses tirées de la forêt ne diffèrent pas significativement chez les pygmées ( $\mathrm{p}=0,345, \mathrm{~F}=1,07$ et $\mathrm{ddl}=2$ ).

- Protection des animaux chez les Batwa : Les os, les poils des animaux sont des éléments aussi très utilisés dans la médecine traditionnelle chez les pygmées. Par prédilection, les ossements du Gorille tenu par une lamelle de peau d'animal autour du cou d'un jeune de moins de 12 ans, permet à l'enfant d'avoir des os durs comme celui de Gorille. Ils utilisent aussi les poils de 
certains animaux pour cicatriser les brûlures. Certains types des pierres sont aussi utilisés pour soigner certaines maladies. Les règles sont riches, et ceux qui leurs désobéissent sont soumis à de sévères punitions : Les «Malambo » fondées sur les valeurs coutumières sont ces endroits sacrés où les animaux mettent bas, et sont strictement interdites pour la chasse. Ils ne mettent pas des cols près des rivières où les animaux vont boire. En cas de désobéissance des règles établies, les gardiens de coutume mettront le «muzombo » sur l'incriminé. C'est une «punition de mort». Que cette mort soit spirituelle ou une excommunication, les membres de la communauté la craignent et respectent avec ferveur les interdits. Dans le Tableau 3, nous donnons les espèces animales protégées par les Batwa ou les Bambuti.

Tableau 3. Les espèces animales protégées par les Batwa ou les Bambuti

\begin{tabular}{ccc}
\hline Nom des animaux & Noms français & Nom scientifique \\
\hline Soko Mutu & Gorilles de Grauer & Gorilla beringei graueri \\
\hline Mboko & Buffle & Syncerus caffer \\
\hline Kikunya Kunya & Potamochère & Tragelaphus scriptus \\
\hline Soko Mutu & Chimpanzé & Pan troglodytes \\
\hline Ingwe & Lion & Panthera leo \\
\hline Njovu & Eléphant & Loxodonta africana \\
\hline Tambwe & Léopard & Panthera pardus \\
\hline Satwe & Piton & Python sebae \\
\hline Ikaga & Pangolin géant & Manis gigantea \\
\hline Ikaga & Petit pangolin & Manis tricuspis \\
\hline Wanjo & Aigle & Aquila wahlbergi \\
\hline
\end{tabular}

Au total, 11 espèces ont été recensées comme espèces totalement protégées par les Batwa. Toutes ces espèces citées dans le tableau 3 ont un taux de réponse élevé [91-100] et sont reconnues par toute la communauté compte tenu de leurs valeurs culturelles. Notons aussi que hormis, le Buffle et le Potamochère les autres espèces citées dans le tableau 3 sont interdites par la Convention sur le Commerce International des Espèces de Faune et de Flore Sauvages menacées d'extinction (CITES).

\section{Peuple Lega des Chefferies de Basile, Lwindi et Wamuzimu}

D'après les conversations avec les gardiens de coutume, «les Lega sont un peuple bantou forestier d'Afrique centrale, établi principalement en RDC, à l'Est du fleuve Congo, de la basse à la haute altitude des monts Mitumba, dans les Provinces du Nord-Kivu, du Sud-Kivu, de la Tshopo et du Maniema. D'après leur histoire, les Lega quittent la région du Nil blanc (Nord-Est de la RDC et Nord-Ouest de l'Ouganda), et plus exactement de la monarchie de "Bunyoro ", au cours du $16^{\text {ieme }}$ siècle pour s'installer sur la rive Est du Lwalaba (fleuve Congo). Ils parlent la langue Kilega et habitent les 
territoires Bulega. Le terme Kilega (Ileka) désigne à la fois la langue et la tradition, la culture et la civilisation du peuple Lega. Avec la colonisation, le Bulega a été morcelé et attribué à plusieurs provinces du Centre-Est de la $R D C »$.

Depuis les crises à répétition consécutives à l'indépendance de la RDC, notre peuple fait partir de ceux qui réclamaient une authentique administration au pays, fondée sur des critères objectifs: cohérence culturelle, démographie satisfaite, étendue adéquate, viabilité économique. L'ambition ultime de l'économie écologique est un bien-être humain durable.

D'après un chef coutumier: "Cela n'inclut pas d'autres considérations telles que l'appui à la restauration du paysage, une justice sociale équitable et intergénérationnelle, une stabilisation de la population et une reconnaissance de la contribution du capital humain Lega et le bien-être humain. Cela passera aussi par un meilleur développement des indices de bien-être». Il continue en disant : «Nous les Balega, respectons les mythes en utilisant les ressources mises à notre disposition par la nature pour divers usages de notre survie. Pour cette raison, nous nous forçons à respecter notre coutume en craignant les sanctions prévues par celle-ci. Partant des sanctions, la coutume lega présente une force dans la gestion de l'environnement».

\section{- Culture et sacralisation}

Chez le peuple lega, le savoir local comprend la maitrise des informations transmisses non écrit des ressources locales, des races animales, des plantes environnante et locales, des cultures et des espèces d'arbres. Il peut comprendre également des informations concernant les associations d'arbres et de plantes qui poussent ensemble, les plantes qui indiquent le niveau de salinité du sol ou celles qui fleurissent au début des pluies.

Sur le plan socioculturel, la forêt est le repère des esprits et le support des représentations mythiques collectives. Les forêts villageoises sont, depuis des temps reculés, objet d'une protection intégrale contre toute exploitation, assurée par les sages des villages. Elle demeure un lieu des ancêtres dont ils en sont nos créateurs. Ils réfugient dans les tombes et différents rites coutumiers.

Le peuple lega est monothéiste, une croyance a « Ombe, Kalaga, ...» malgré cette croyance, ce peuple fait le recours à certaines forces invisibles selon le besoin, par exemple pour la naissance, la fertilité des terres, pour faire une bonne chasse ou pêche,... La culture Lega est centrée sur la solidarité réciproque et sur l'amour du prochain.

Au moment du départ pour le rite, qui se déroule dans la forêt, les jeunes garçons, dont l'âge varie entre 14 et 17 ans, doivent se déshabiller et porter un simple linge pour se couvrir le sexe. A la date de départ pour l'usage 
rituel, la fête est organisée dans tous les villages. Pendant la période du rite, c'est «Kimbilikiti» ou l'esprit qui est l'autorité souveraine interdit toute activité dans la forêt sacrée où se passe l'apprentissage. Le rite «Bwali » à côté de «Nyano» pour les filles reste mystique et ésotérique, le jour qui précède l'initiation.

\section{Interdits et sanctions sectorielles chez les Lega}

Suivant la légende, les éléments culturels tels que la valeur, les normes, les bien êtres, les techniques coutumières ont été transmis par les aïeux et seront légués aux générations futures. Tous les membres de la communauté sont obligés de respecter et se soumettre à l'autorité et aux milieux sacrés car chez ce peuple, la pluralité des normes évoque la particularité de leur coutume. Le respect des normes établies est d'une importance capitale pour ne pas subir des sanctions graves.

Par exemple, les arts lega qui regorgent des trésors historiques, culturel sont respectés suivant sa diversité. Une très grande diversité artistique y est constatée: la sculpture, le statuaire, la vannerie, le tissage, l'esthétique du corps, les armes blanches etc.

\section{Genres}

Chez les Lega, la femme est une gestionnaire des produits forestiers apportés par son époux, dans la gestion du foyer et est considérée comme une conseillère familiale. Elle assure la formation des jeunes filles sur la coutume et intervient dans l'initiation des jeunes filles appelée «Boumbwa». Elle n'intervient en aucun travail dans la forêt sauf à la recherche des certains champignons comestibles, la recherche des plantes médicinales, du bois de chauffage.

La femme n'a pas le droit de se mettre dans la cour royale car là il n'y a aucun mot ni la parole. Certaines d'entre elles ont aussi des places dans des solennités royales lors de l'investiture d'un nouveau chef coutumier.

\section{Pratique et savoir locaux chez les Lega}

-Protection du monde végétal artistique: La vannerie ou l'art de tresser les fibres végétales est très présente et utilisée pour des objets très variés (paniers, filets traditionnels, corbeilles, mandes, vans, meubles, nattes, lits etc.). Ils sont utilisés rationnellement pour servir aussi les générations postérieures. C'est ainsi que la coutume lega intervient beaucoup dans la gestion de biotope tout en faisant respecter les règles établies par les ancêtres et la coutume (Tableau 4). 
Tableau 4. Les espèces souvent utilisées pour les vanneries mais avec usage rationnel

\begin{tabular}{cc}
\hline Noms vernaculaires & Noms Scientifiques \\
\hline Ibondo, ibila & Eleais guinnéesis \\
\hline Kekele & Eremospatha haullevilleana \\
\hline Mutondo ou lububi & Eremospatha macrocarpa \\
\hline Raphia, lweku, yamwasamusa & Raphia gilletii \\
\hline Makungu & Megaphrynium macrostachysum \\
\hline
\end{tabular}

Cette protection est basée sur l'interdiction par les sages et les membres de la cour royale qui pratiquent la mythologie et le rituel pour empêcher la destruction de la biodiversité. Les essences productives des chenilles et d'autres espèces protégées sont strictement interdites à la coupe par la coutume Lega. Par exemple : Ficus elastica « Kilondolondo » qui sert dans la fabrication des vêtements coutumiers chez les gardiens de coutume ; Uapaca benguelensis est utilisée au traitement des maladies sorcières appelées «Milongé » et Maesa lacealanta «Bwaga » est utilisée pour chasser les démons d'origine conflictuelle. Ces consignes sont respectées par l'ensemble des Lega (Tableau 5 et 6). Celle-ci n'a pas changé et est pratiquée par les gardiens des coutumes et les communautés locales ».

Tableau 5. Les espèces végétales protégées par la coutume Lega

\begin{tabular}{c|c}
\hline Noms vernaculaire lega & Noms Scientifiques \\
\hline Mushishi & Cynometra hankei \\
\hline Kashebeye & Albizia adianthifolia \\
\hline Ibungulu (Bulungu) & Autranella congolensis \\
\hline Mukusu & Canarium schweinfurthii \\
\hline Kabungu & Diospyros bipindensis \\
\hline Kabungu & Diospyros zenkeri \\
\hline Bwaga (mwanga) & Maesa lacealanta \\
\hline Lukundu & Piptadeniastrum africanum \\
\hline Achukwe-chukwe & Prunus africana \\
\hline Katondo & Rauwolfia vomitoria \\
\hline Kyanina-kya-Kabi & Struchnos memecyloides \\
\hline Kyanina-kya-Kabi & Struchnos scheffleri \\
\hline Musela & Uapaca benguelensis \\
\hline Kilondolondo & Ficus elastica \\
\hline
\end{tabular}

Parmi ces 14 espèces citées dans le Tableau 5, certaines comme Canarium schweinfurthii, Prunus africana, Ficus elastica, Piptadeniastrum africanum, Uapaca benguelensis et Maesa lacealanta ont chacune un taux de réponse respectif de $79-100 \%$. Elles sont reconnues par divers âges. Ces arbres sont gardés pour leur utilité comme: l'ombre pour le repos et la prise de repas; leurs feuilles mortes, l'attraction et le repos des animaux sources de déjections fertilisantes, les fruits consommés et vendus, l'utilisation de leurs 
organes dans la pharmacopée. Certaines autres espèces végétales comme Kambaya, Wambu, Ambilombilo sont les plus médicinales et plus discrètes par la coutume. Nous n'étions pas autorisés d'en avoir les échantillons.

- Protection des animaux : Chez le Lega, la protection des certaines animaux n'est pas un fait du hasard. D'après leur coutume, après la création, l'utilité de certaines plantes utiles a été révélée aux hommes par les animaux ou les génies à travers des songes. C'est ainsi que le symbolisme animal dévoile certains animaux comme des héros civilisateurs de l'humanité (peau de lion, léopard, plume d'aigle, portés par le chef coutumier), en ce sens que dans les mythes fondateurs, il est interdit de les tuer. Dans le Tableau 6 présente la liste des animaux interdits de tuer par la coutume lega.

Tableau 6. Les espèces animales protégées par la coutume lega

\begin{tabular}{|c|c|c|}
\hline Nom des animaux & Noms français & Nom scientifique \\
\hline Soko mutu & Gorilles de Grauer & Gorilla beringei graueri. \\
\hline Mubale & Antilope & Neotragus batesi \\
\hline Mboko & Buffles & Syncerus caffer \\
\hline kikunya kunya & Potamochères & Tragelaphus scriptus \\
\hline Soko mutu & Chimpanzés & Pan troglodytes \\
\hline \multirow[t]{6}{*}{ Quelques singes } & Colobe d'Angola & Colobus angolensis \\
\hline & Colobe rouge & Piliocolobus oustaleti \\
\hline & Cercopithèque de Brazza & Cercopithecus lhoesti \\
\hline & Singe (Colobe) bleu & Cercopithecus mitis \\
\hline & Colobe à queue rouge & Cercopithecus ascanius \\
\hline & Cercopithèque en dent & Cercopithecus denti \\
\hline Ingwe & Lion & Panthera leo \\
\hline Ngwenna & Crocodile & Crocodylus niloticus \\
\hline kivulovulo & Crapaud géant & Rhinella marina \\
\hline Ikaga & Pangolins géants & Manis gigantea \\
\hline Ikaga & Petit pangolin & Manis tricuspis \\
\hline Njovu & Eléphants & Loxodonta africana \\
\hline Wanjo & Eigle & Aquila wahlbergi \\
\hline Tambwe & Léopard & Panthera pardus \\
\hline Njuki & Abeilles & Halictidae \\
\hline satwe & Piton & Python sebae \\
\hline Nkomo & Poissons & - \\
\hline Nsamba & Poisson chat africain & Clarias $s p$ \\
\hline
\end{tabular}

Au total, 23 espèces animales sont été recensées et protégées par la tribu Lega. Sauf le Poisson chat africain, Antilope, Buffles Potamochères, les autres espèces protégées par la coutume lega sont défendues par CITES. Toutes ces espèces citées dans le Tableau 7 ont un taux de réponse élevé et reconnus par toutes les personnes matures compte tenu de leurs valeurs 
culturelles. Malheureusement, certains animaux comme Colobus angolensis, Piliocolobus oustaleti, Cercopithecus lhoesti, Cercopithecus mitis, Cercopithecus ascanius, Cercopithecus denti, Clarias sp., Neotragus batesi, Syncerus caffer, Tragelaphus scriptus sont consommés par les nouvelles générations d'une manière clandestine ou soit tués et vendus dans d'autres tribus consommatrices.

\section{Éthique et interdiction coutumière chez le peuple Lega}

La coutume Lega se présente sous une forme de principes généraux, de notions fondamentales, de préceptes et d'interdits d'ordre religieux, moral et social, qui s'expriment dans les différentes institutions hiérarchiques de ce peuple. Ces normes définissent la modalité d'exploitation des ressources de la forêt, ils protègent aussi les communautés et insistent dans la sauvegarde des leurs lois et coutumes qui ne peuvent changer à n'aucun cas.

Parfois, on se réfère simplement à la variété des normes de légitimité ou de légitimation qui règlent les diverses sources au niveau du pouvoir et d'autorité traditionnelle. Partant de la coutume lega, des faute graves sont soumises à des sanctions qui peuvent être fatales à l'endroit des fautifs et même de toute sa famille. Notons que, la coutume lega prévoit plusieurs sanctions selon les types d'interdits que l'on énumère dans les Tableaux (7 et 8).

D'autres interdictions sont comme l'usage abusif de l'eau car cela peut entrainer le l'arthrite du pied (gonflement des pieds) jusqu'à la mort. Le nonrespect des sites sacrés sont tributaires à la mort.

Tableau 7. Les animaux interdits et sanctions sectorielles chez les Lega

\begin{tabular}{|c|c|c|}
\hline Interdits & Sanctions & Motif d'interdiction \\
\hline $\begin{array}{l}\text { Panthera pardus } \\
\text { (Léopard) }\end{array}$ & La mort & $\begin{array}{c}\text { Sa peau est utile dans la fabrication des } \\
\text { ustensiles royale et intervient pour signifier le } \\
\text { grade d'un gardien de coutume. }\end{array}$ \\
\hline $\begin{array}{l}\text { Panthera leo } \\
\text { (Lion) }\end{array}$ & $\begin{array}{l}\text { La mort des tous les } \\
\text { membres de la } \\
\text { famille. } \\
\end{array}$ & $\begin{array}{l}\text { Sa peau et sa queue sont utilisées dans la } \\
\text { tradition et sa queue }\end{array}$ \\
\hline $\begin{array}{c}\text { Manis gigantea } \\
\text { Le pangolin géant }\end{array}$ & La mort & $\begin{array}{l}\text { Sa peau intervient dans la fabrication du } \\
\text { chapeau du grand Roi appelée «Bewa» }\end{array}$ \\
\hline $\begin{array}{l}\text { Crocodylus niloticus } \\
\text { Le crocodile }\end{array}$ & La mort & Son pancréas produit du poison \\
\hline $\begin{array}{l}\text { Aquila wahlbergi } \\
\text { Aigle }\end{array}$ & La mort & Ses griffes symbolisent le grand du Roi. \\
\hline $\begin{array}{c}\text { Gorilla beringei graueri } \\
\text { Le gorille }\end{array}$ & La mort & $\begin{array}{c}\text { Ses os sont utilisés pour donner la force aux } \\
\text { gardiens de la coutume. }\end{array}$ \\
\hline $\begin{array}{c}\text { Loxodonta africana } \\
\text { L'éléphant }\end{array}$ & La mort & $\begin{array}{c}\text { Un héritage sacré pour la coutume et la famille } \\
\text { royale }\end{array}$ \\
\hline Nkomo & La mort & Ses dents symbolisent un grade quelconque \\
\hline Nsamba & La mort & $\begin{array}{l}\text { Sa peau est utilisée pour la fabrication des } \\
\text { chapeaux. }\end{array}$ \\
\hline
\end{tabular}


Tableau 8. Les végétaux interdits et sanctions sectorielles chez les Lega

\begin{tabular}{|c|c|c|}
\hline Interdits & Sanctions & Motif d'interdiction \\
\hline $\begin{array}{l}\text { Canarium schweinfurthii } \\
\text { (Musuku) }\end{array}$ & $\begin{array}{c}\text { Nourrir les gardiens en cas de } \\
\text { non-respect, le contre venant } \\
\text { subit la peine de mort }\end{array}$ & $\begin{array}{l}\text { Produit du feu sous forme de } \\
\text { bougie. }\end{array}$ \\
\hline $\begin{array}{l}\text { Maesa lacealanta } \\
\text { (Bwaga) }\end{array}$ & $\begin{array}{c}\text { Nourrir les gardiens en cas de } \\
\text { non-respect, le contre venant } \\
\text { subit la peine de mort }\end{array}$ & $\begin{array}{l}\text { Sert à chasser les démons et ales } \\
\text { invites s'il le faut. }\end{array}$ \\
\hline $\begin{array}{l}\text { Autranella congolensis } \\
\text { (Ibungulu) }\end{array}$ & Exterminer toute la famille & $\begin{array}{l}\text { Sa peau contribue à la fabrication } \\
\text { des choses mystiques pour le Roi. }\end{array}$ \\
\hline $\begin{array}{l}\text { Ocotea unsambariensis } \\
\text { (Licheche) }\end{array}$ & $\begin{array}{c}\text { Nourrir les gardiens en cas de } \\
\text { non-respect, le contre venant } \\
\text { subit la peine de mort }\end{array}$ & Produit des planches. \\
\hline $\begin{array}{l}\text { Uapaca benguelensis } \\
\text { (Musela) }\end{array}$ & $\begin{array}{c}\text { Nourrir les gardiens en cas de } \\
\text { non-respect, le contre venant } \\
\text { subit la peine de mort }\end{array}$ & $\begin{array}{c}\text { Sert à traiter des maladies graves } \\
\text { comme des « Milongé » et agit dans } \\
\text { la signification des divorces }\end{array}$ \\
\hline (Kahabi) & $\begin{array}{c}\text { Nourrir les gardiens en cas de } \\
\text { non-respect, le contre venant } \\
\text { subit la peine de mort }\end{array}$ & $\begin{array}{l}\text { Traite des mots des ventres et des } \\
\text { plaies très graves. }\end{array}$ \\
\hline (Mbilombilo) & $\begin{array}{c}\text { Nourrir les gardiens en cas de } \\
\text { non-respect, le contre venant } \\
\text { subit la peine de mort }\end{array}$ & $\begin{array}{l}\text { Ses graines sont comestibles et sont } \\
\text { utilisées par les hommes et les } \\
\text { animaux. }\end{array}$ \\
\hline Musitu & $\begin{array}{c}\text { Nourrir les gardiens en cas de } \\
\text { non-respect, le contre venant } \\
\text { subit la peine de mort }\end{array}$ & Liane produisant de l'eau. \\
\hline $\begin{array}{l}\text { Ficus elastica } \\
\text { Kilondolondo }\end{array}$ & $\begin{array}{c}\text { Nourrir les gardiens en cas de } \\
\text { non-respect, le contre venant } \\
\text { subit la peine de mort }\end{array}$ & $\begin{array}{c}\text { Sert dans la fabrication des } \\
\text { vêtements surtout chez les Batwa. }\end{array}$ \\
\hline
\end{tabular}

\section{La Gestion Environnementale Et Savoirs Coutumiers Valorisation des Savoirs Traditionnels}

Contrairement aux stratégies traditionnelles de type collectif basées et maintien des savoirs locaux sur la conservation des aires protégées, certains auteurs comme (Omari, 1990; Byers, 1997; Omari et al., 1999; Houngnihin 2005; Mangambu et al., 2017) ont montré que la gestion des ressources biologiques et géologiques basée sur les méthodes de conservation modernes ont démontré les limites dans les pays de l'Afrique subsaharienne en général et dans le bassin du Congo en particulier. Ainsi, le retour vers les savoirs locaux est devenu un idéal et une des solutions adéquates (Houngnihin, 2005; Langewiesche, 2006; Sanou, 2010).

L'implication de la population locale dans la gestion des écosystèmes dévient alors une nécessité impérieuse (Sene et al., 2013). Elle est à la base des engagements et des diplomaties de conservation de l'environnement et la Convention sur la Diversité Biologique (CDB), en liant la divergence culturelle à la diversité biologique. Ceci a permis aux «populations autochtones et locales » de faire entendre leur voix et d'accéder à une certaine 
reconnaissance sur la dispute internationale dans le cadre de la gestion de la biodiversité (Langewiesche, 2006).

Impliquer et responsabiliser une communauté villageoise riveraine dans l'aménagement et la gestion d'une forêt nécessite que l'on s'inspire de sa tradition qui est un jalon, un cadre de l'avis et de ses habitudes qui sont une mise en œuvre de la coutume et une disposition sacralisée par des rêves des aïeux (Langewiesche, 2006).

Cette démarche est d'actualité dans un monde à la recherche de nouveaux repères où chaque société tente de s'auto représenter dans une contrariété de meilleur d'elle-même en construisant un monde à visage plus humain et ayant le souci du quotidien des générations ultérieures (Sanou, 2010). Ainsi, l'implication de l'intégration des savoirs endogènes de la gestion de la diversité biologique n'est pas incompatible avec l'amélioration des moyens d'existence aux alentours des aires protégées. Mais aujourd'hui, les vestiges de cette tradition ancestrale qui sont sacrés de ses savoirs endogènes ou locaux témoins des produits forestiers, sont de plus en plus menacés par la forte densité et croissance démographique et l'exploitation des produits forestiers non lignés (Mubalama et al., 2018).

D'après Cousin et Martineau (2009) et Mangambu et al. (2015), les Us et coutumes autours des aires protégées représentent des symboles identitaires des différentes sociétés. Chez les pygmées et les peuples Lega, la forêt et ses composantes sont des endroits sacrés et offrent à la population des avantages variés et divers. Ces peuples sont des conservateurs des pratiques traditionnelles. La tradition confère à la communauté villageoise son identité et sa personnalité et lui donne la capacité de relever les grands défis qui se présentent à chaque époque (Sanou, 2010).

Pour cette raison, nous pensons que les savoirs locaux des peuples étudiés sont alors devenus pour ces peuples l'objet d'enjeux politiques de conservation sur les modes de gestion traditionnels de la nature et sont forcément créateurs de la biodiversité et peuvent servir d'outils pour les politiques contemporaines de conservation dans les aires protégées. Donc, pour conserver la biodiversité, nous devons comprendre comment les cultures humaines interagissent avec les paysages naturels et les transforment en paysages culturels.

\section{Protection de la Biodiversité par les Structures Traditionnelles de deux Tribus}

Les complexes forestiers constituent au sud du Sahara des espaces munis des droits et d'usages coutumiers (Yombatina, 2000 ; Akouehou, 2004). Dans la région du Kivu, depuis l'impérialisme des colonisateurs, la culture traditionnelle s'est nettement affaiblie et les opinions ont changé dans certaines communautés. A la grandeur des facteurs qui expliquent cette 
mutation, il y a la pression humaine exercée sur les ressources dues à l'explosion démographique et le comportement des agents de l'administration publique qui ont parfois une certaine réserve par rapport aux valeurs culturelles (Mangambu et al., 2015a). C'est la même chose qui se passe chez les Lega et les Pygmées vivant dans les environs de la RNI.

D'après Prigogine (1985), la protection de la faune sauvage et son habitat naturel étaient jadis protégés dans le terroir forestière d'Itombwe par les communautés environnantes. Cette protection était basée surtout sur des Us et coutumes ou sur lerite et contribuait à limiter la prédation. Ces dernières années des nouvelles visions chrétiennes sont venues rompre la tradition sur l'exploitation rationnelle de la biodiversité. Beaucoup d'espèces animales interdites font aujourd'hui l'objet d'une vente illicite (Omari et al., 1999).

Nos investigations montrent que chacune de tribus devient partie prenante de la gestion des forêts et des principes d'usage. Chacun de ces groupes tribaux appartient à des groupes différents qui structurent leurs actions en fonction des systèmes de production et de représentations sociales.

Dans l'ensemble, suivant l'interdiction, le peuple Lega enregistre beaucoup d'interdictions coutumières que les Batwa, mais le peuple pygmée garde encore l'identité coutumière. Et la majorité des espèces tant animales que végétales sont protégées par l'Union Internationale pour la Conservation de la Nature1le (UICN) qui est l'une des principales organisations non gouvernementales mondiales consacrées à la conservation de la nature des environnements vulnérables, menacés ou en danger (Juhé-Beaulaton, 2008), chez le peuple Lega, l'importance des interdits et des règles coutumières dans la conservation

\section{Les Statuts Juridiques Traditionnels Légués par leurs Aïeux}

Les statuts juridiques coutumiers déterminent les lois et les devoirs à la conservation de la nature léguée par les aïeux. Ce modèle s'appuie sur une organisation collective coutumière qui sert de moteur aux activités de surveillance et de monitoring sur la conservation de la nature.

D'après les Chefs des chefferies, la régulation de ces procédures pourrait conduire à la conservation de la naturelle si l'autorité publique le réglemente par une loi, malheureusement la forte croissance démographique et l'insuffisance des infrastructures médicales constituent des problèmes majeurs auxquels se heurtent les Lega.

A cet effet, les nouvelles générations, en complicité avec les parents, font toujours recours aux produits forestiers non lignés pour satisfaire les besoins primaires en abandonnant la tradition et la coutume créant ainsi les crimes directes et atteintes aux biens naturels et au développement durable. 


\section{Enjeux à la Conservation de la Biodiversité}

Notons que l'initiative du début des années 1990 et 1996 sur le financement de la conservation du massif d'Itombwe avait l'accéléré des réflexions et des outils relatifs à la conservation de la biodiversité de ce massif. En effet, durant l'intervalle de cette période, un mouvement international largement porté par les grandes ONG de conservation intervenant en milieu tropical (Wildlife Fund of Nature, Wildlife Conservation Society) réfléchit sur les modalités de financement de cette aire protégée.

Par ailleurs, cette période est caractérisée par une remise des projets de conservation et de développement intégré, qui constituent jusqu'alors le fer de lance des politiques de conservation en milieu pauvre qui était protégé par les structurelles locales, et des Us et coutumes. Le bilan que de nombreux acteurs font à cette époque est que le soutien à des activités génératrices de revenus permettant d'augmenter les recettes issues d'activités durables a un impact limité.

\section{Facteurs de Dégradation de l'Environnement de la RNI}

La RNI est classée dans la catégorie VI des aires protégées selon la catégorisation de l'UICN et regorge trois zones, à savoir la zone de conservation intégrale, la zone tampon et la zone à usage multiple. Cette dernière compte, à elle seule $44,9 \%$ de la Réserve, mais la population attaque aussi d'autres zones (PAG RNI, 2017; Mangambu, 2021).

Parmi ces objectifs de gestion on compte la protection de maintien à long terme de la diversité biologique et des autres valeurs naturelles du site, la promotion des pratiques rationnelles de gestion, enfin l'assurance d'une productivité durable consistant à protéger le capital de ressources naturelles contre toute aliénation engendrée par d'autres formes d'utilisation du sol susceptibles de porter préjudice à la diversité biologique de la région et de contribuer au développement régional et national. Par ailleurs, malgré ces objectifs, les Us et coutumes, dans cette réserve, on constante l'absence d'une gestion participative, l'absence des plans d'affectation des terres, la faiblesse des capacités nationales et l'inadaptation des lois et règlements forestiers aux réalités sociologiques, culturelles et économiques de la population riveraine.

Cet état de chose pousse la réserve à régresser et ne pas être à même d'assurer pleinement la mission lui assignée. Malgré les systèmes d'interdits, de nombreux problèmes apparaissent autour de la Réserve Naturelle d'Itombwe. Ces problèmes concernent essentiellement deux points :

- la diminution de la superficie de l'espace occupé par la population locale, provoquée par la forte pression sur les terres ;

- la surexploitation des ressources de la forêt jusqu'à toucher les espèces interdites. 
Les piégeages pour avoir les gibiers satisfaisant aux besoins quotidiens des familles, les peaux des animaux protégés vendus très chères (exemple: Panthera pardus, Panthera leo, Manis gigantea, Gorilla beringei graueri, Crocodylus niloticus, plume Aquila wahlbergi...), les plantes médicinales (Prunus africana, Rauvolfia sp, Struchnos memecyloides, Lebrunia bushaie, Prunus africana, Kigelia africna), des coupes clandestines des bois d'œuvres (Canarium schweinfurthii, Uapaca benguelensis, Piptadeniastrum africanum, Albizia adianthifolia), l'exploitation des minerais devient de plus en plus grandissante malgré les interdits traditionnels, et cela par la forte demande des bois de chauffe, surtout en milieu urbain. D'après les études menées par Aruna (2015), les énergies traditionnelles (bois et charbon de bois par exemple) représentent $89 \%$ de la consommation des ménages ruraux et urbains.

L'introduction de nouvelles confessions religieuses chrétiennes (surtout les églises de réveil) dont certaines idéologies s'opposent fortement aux pratiques des rites traditionnels jugés sataniques et démoniaques, a aussi ébranlé la cohésion sociale et contribué à la fragilisation des forêts sacrées et des conversations, menaçant ainsi leurs existences. Cela tient du fait que l'établissement et la protection des forêts du massif d'Itombwe étaient principalement basés sur des croyances culturelles et religieuses locales.

Donc, la régression des pratiques ancestrales liées aux savoirs culturels et conservation de la biodiversité chez les peuples Pygmée et Lega riverains de la Réserve Naturelle d'Itombwe peuvent cependant, avoir d'énormes conséquences pour la société, notamment rurale. Ainsi, cette étude, montre que l'avis de certains Lega et Batwa, rime avec l'abandon des traditions ancestrales pour satisfaire leurs besoins suite aux deux points évoqués dans ce paragraphe.

\section{Conclusion}

L'usage et l'importance des Us et coutumes constituent, sans doute, des lieux de refuge pour des nombreuses espèces dans le cadre de conservation de la nature. En effet, les interdits coutumiers et la perception propice qu'ont les habitants quant au caractère mystique de certaines espèces sont des atouts pour une bonne protection que la conservation ressources naturelles locales en général.

Chez Batwa, les systèmes traditionnels de gestion de la biodiversité sur les Us et coutumes révèlent d'une importance particulière sur le caractère sacré, confidentiel et même secret d'une partie de ces connaissances chez ces peuples. Certains endroits comme Les « Malambo » sont des biotopes sacrés, 17 espèces végétales et b11 espèces animal ont été recensées comme espèces totalement protégées et 14 espèces végétales sont d'usage rationnel.

Chez l'autre peuple Lega, il y a des éthiques et des interdictions sanctions sectorielles qui peuvent amener à la mort. D'autres interdictions sont 
comme l'usage abusif de l'eau car cela peut entrainer le l'arthrite du pied (gonflement des pieds) jusqu'à la mort. Le non-respect des sites sacrés sont tributaires à la mort. Pour ce peuple, 23 espèces animales sont été recensées et protégées et 14 espèces végétales sont totalement interdites suivant mythologie Lega.

Mais avec la contrainte foncière présente autours des aires protégées et les mutations socioculturelles induites par la forte implantation du christianisme, a fragilisé des interdits ancestraux, des principes et des fétiches impliquant la préservation des espèces et leurs habitats naturels. Ce qui expose les ressources naturelles du massif d'Itombwe à une exploitation anarchique, malgré l'usage des interdictions coutumières Lega et Batwa d'Itombwe.

Pour sauvegarder la biodiversité du massif malgré les Us et coutumes et les pressions anthropiques, il est alors nécessaire d'entreprendre certaines actions : (i) les acquis de la législation coutumière dans la législation moderne de conservation de l'environnement et (ii) préserver et mettre l'accent sur les traditions tout en ayant à l'esprit que les connaissances locales des Us et coutumes maintiendront la biodiversité sans enfreindre la production locale mais il est pourtant important de s'en inspirer en innovant des stratégies efficaces.

Dans l'optique de la conservation de la biodiversité, il nous semble donc important de déployer des efforts pour protéger certains types d'attitudes culturels car chaque communauté locale prend des décisions sur la façon d'utiliser les ressources naturelles de son environnement dans le cadre de son propre choix de valeurs. Les décisionnaires doivent prendre en compte ces valeurs et les pratiques qui en découlent, pour un usage durable de la biodiversité.

\section{Remerciements}

Les auteurs expriment leur gratitude envers la World Wildlife Fund of Nature (WWF) pour son aide scientifique et son appui financier à cette recherche et l'Institut Congolais pour la Conservation de la Nature (ICCN), les autorités locales et d'autres partenaires pour leurs collaborations.

\section{References:}

1. Agarwal, B. (2009). Rulemaking in community forestry institutions: The difference women make. Ecological Economics, 2296-2308.

2. Akouehou, G.S. (2004). Environnement institutionnel et gestion traditionnelle des espaces forestiers: cas de la région des Monts Kouffé au Centre du Bénin. Cahier d'autre mer, 175-190, https://doi.org/10.4000/com.526

3. Arrey, W.H. \& Loumou-Mondoleba, A-P. (2021). Les Politiques Publiques De Gestion Des Dechets Au Cameroun: Une Analyse 
Critique Du 'Monopole' D'hysacam A L'ere De La Gouvernance Decentralisee Dans La Ville De Yaounde. European Scientific Journal, ESJ, 17(15), 430. https://doi.org/10.19044/esj.2021.v17n15p430

4. Bikaba, D. (2013). Peuples autochtones, Communautés locales et Politique nationale : vers les objectifs d'AICHI de la CDB en République Démocratique du Congo, rapport, $15 \mathrm{p}$.

5. Bisidi, Y., Languy, M., Baleke, E., Lusuna, M., Muhigwa, B., Bisusa, G. \& Basabose, A.K. (2008). L'utilisation et la conservation des ressources forestières à l'Itombwe : perception de la population. WWF EARPO 1-54p.

6. Bouzou. M. \& Boubacar, Y. (2008). Savoirs locaux et gestion des écosystèmes sahéliens. Les Cahiers d'Outre-Mer, 241-242

7. Butare, I. (2003). Pratiques culturelles, La sauvegarde et la conservation de la biodiversité en Afrique de l'Ouest et du Centre Actes du Séminaire-Atelier de Ouagadougou (Burkina Faso), du 18 au 21 juin 2001. Centre de recherches pour le développement international, $280 \mathrm{p}$

8. Byers, B. (1997). Démarche pour comprendre et influencer les comportements à l'égard de la conservation et de la gestion des ressources naturelles. Washington D.C. : Programme d'appui à la biodiversité, Bulletin sur la biodiversité africaine, numéro 4,

9. Colchester, M. (2003). Nature sauvage, nature sauvée ? Peuples autochtones, aires protégées et conservation de la biodiversité. Forest Peoples Programme,

10. Coulibaly, P.B. (1995). Rite et société à travers le Bafili: Une cérémonie d'initiation à la géomancie chez les Bambara du Mali. Ed Jamana, Bamako.

11. Doumenge, C. \& Schilter, C. (1997). Les Monts Itombwe. D'une enquête environnementale et socio-économique à la planification d'interventions au Zaïre. UICN, Brazzaville, Congo \& IZCN, PIL et SIDEM, Bukavu, Zaïre: XII + 269 p.

12. Kohler, F. (2012). Diversité culturelle et diversité biologique : une approche critique fondée sur l'exemple brésilien. Natures Sciences Sociétés 2 (19), 113 -124

13. Greenbaum, E. \& Chifundera, K. Z. (2012): Conservation implications following the rediscovery of four frog species from the Itombwe natural reserve, eastern Democratic Republic of the Congo. Herpetological Review 43, 253259.

14. Hart, J. \& Mubalama, L. (2005). Conservation of gorillas and chimpanzees in the Itombwe Massif. In Gorilla Journal of Berggorilla \& Regenwald Direkthilfe No. 30, pp 7-8 
15. Hounghihin, AR. (2005). Les Mécanismes Endogènes dans la Problématique del'Environnement à Covè (Benin).CODESRIA : Maputo (Mozambique) ; 1-18.

16. Juhé-Beaulaton, D. (2008). Sacred forests and the global challenge of biodiversity conservation: the case of Benin and Togo. Journal for the Study of Religion, Nature, and Culture: 351-372. https:// hal.archivesouvertes.fr/halshs-00090462/document

17. KIMONI, Iyay (1975). Destin de la littérature négro-africaine ou problématique d'une culture, Kinshasa : Presses universitaires du Zaïre ; Ottawa : Editions Naaman, 273 p..

18. Kingsbury, B. (1996). The Concept of Indigenous Peoples in Asia: International Law Issues. In: Christian Erni (éd.) Vines that Bind. International Workgroup for Indigenous Affairs, Copenhague: 53-73.

19. Kyale, K. \& Maindo, M. (2017). Pratiques Traditionnelles de Conservation de la Nature à L'épreuve des Faits Chez Les Peuples Riverains de la Réserve de Biosphère de Yangambi (RDC). European Scientific Journal, 13 (8) : 328-256

20. Langewiesche, K. (2006). La forêt, les ancêtres et le marché. Perceptions locales de la forêt et de ses changements au Nord-Bénin. Afrika Spectrum, 2(41): 221-248.

21. Mangambu, M. \& Kambale, K. (2019). Pratiques traditionnelles et culturelles des communautés locales en matière de la conservation de la biodiversité : cas dans la Réserve Naturelle d'Itombwe à l'Est de la RD Congo Network, Workhop Bukavu, 1-23 p

22. Mangambu, M. (2021). État des lieux sur la Biodiversité et la Situation Socio-Economique, de Bassin Nord du Lac Tanganyika : Sud-Kivu, République Démocratique du Congo. (Gestion Durable, Sécurité des Terres et des Eaux). Rapport, Union Internationale de la Conservation de la Nature et Fonds Mondial pour la Nature, 191p

23. Mangambu, M. (2016). Diversité, Biogéographie et Ecologie des Ptéridophytes : Cas de massif montagneux du Parc National de Kahuzi-Biega à l'Est de la RD. Congo. Editions universitaires européennes, $354 \mathrm{p}$.

24. Mangambu, M., Diggelen, R., Mwanga Mwanga, J. C., Ntahobavuka, H., Malaisse, F. \& Robbrecht, E. (2012). Étude ethnoptéridologique, évaluation des risques d'extinction et stratégies de conservation aux alentours du Parc National de Kahuzi Biega (RD Congo). Revue GeoEco-Trop, 36 : 137-158.

25. Mangambu, M., Aluma, K., van Diggelen, R., Rugenda-Banga, R., Mushangalusa, K., Chibembe, S., Ntahobavuka, H., Nishuli, B. \& Robbrecht, E. (2015a). Etudes ethnobotanique et ethnolinguistique des ressources forestières ligneuses utilisées par la population du couloir 
écologique du parc national de Kahuzi-Biega (R D. Congo). European Scientific Journal, 11 162,http://dx.doi.org/10.19044/esj.2014.v.183

26. Mangambu, M., Muyisa, K., Nishuli, B.R. \& Ntahobavuka, H. (2015b). Utilisation des ressources forestières ligneuses par la population habitant la zone submontagnarde du Parc National de Kahuzi-Biega (R.D. Congo). International Journal of Innovation and Applied Studies, $11 \quad$ (2): 508-521. http://ijias.issrjournal.org/abs.php?article=IJIAS-15-094-09

27. Mangambu, M., Ntahobavuka, H., Basinyize, B., Lokumu, I. \& Robbrecht, E. (2018). Pteridaie: indicator of the vegetation succession and dynamics in the forests Chablis and Edges in Kahuzi-Biega National Park (Eastern D. R. Congo, Albertine Rift). International Journal of Biological Research, 6 (1) 5-13, http://dx.doi.org/10.14419/ijbr.v6i1.9241

28. Maregesi, S., Ngassapa, O., Pieters, L. \& Vlietinck, A. (2007). Ethnopharmacological survey of the Bunda district, Tanzania: Plants used to treat infectious diseases. J. of Ethnoph., 113: 457-470.

29. Maretti, CI. (2003). Protected Areas and Indigenous and Local Communities in Brazil: Lessons Learned in the Establishment and Management of Protected Areas by Indigenous and Local Communities in South America. IUCN (Brésil).

30. Marlise, S.K.B., Mbassi, T.A., François, M.E., Doris, N.J.N. \& Touoyem, F.M. (2021). Le marché des produits vivriers et développement socio-économique dans l'Arrondissement de Sa'a (Région du Centre, Cameroun). European Scientific Journal, 17(16), https://doi.org/10.19044/esj.2021.v17n16p72

31. Mboa, N. (2015). « Stratégies de valorisation des savoirs locaux africains : questions et enjeux liés à l'usage du numérique au Cameroun ", Éthique publique [En ligne], vol. 17, $\mathrm{n}^{\circ} 2$ : http://journals.openedition.org/ethiquepublique/2343 ;

32. Merceron, T. (2011). Les mécanismes de gestion de l'environnement en Haïti : Pour une intégration des pratiques traditionnelles, Mémoire de Master, Université Senghor, Alexandrie.

33. Mubalama, K., Igunzi, A., Banswe, T., Asesa, L. \& Kambale, K. (2018). Savoirs traditionnels conciliés aux connaissances scientifiques comme nouveau paradigme de la conservation des Aires Protégées : Cas de Malambo (Réserve Naturelle d'Itombwe, RD Congo). Annales des Sciences et des Sciences Appliquées, vol. 4(3), 129-167

34. Narcisse, M. \& Prudent, R. (2010). Situation de la médecine traditionnelle en Haïti, État des lieux, Rapport d'Études du 
Programme d'Appui au Développement du Système de Santé, Port-au Prince.

35. Omari, I., Hart, J., Butynski, T.M., Birhashirwa, N.R., Upoki, A., M'Keyo, Y., Bengana, F., Bashonga, M. \& Bagurubumwe, N. (1999). The Itombwe Massif, Democratic Republic of Congo: biological surveys and conservation, with an emphasis on Grauer's gorilla and birds endemic to the Albertine rift. Oryx 33, 301-322.

36. Omari, C.K. (1990). "Traditional African Land Ethics." Dans Ethics of Environment and Development: Global Challenge, International Response. Engel, J. Ronald, et Joan Gibbs Engel, éd. 1990. Tucson, Ariz.: University of Arizona Press. pp. 167-175

37. Plan d'Aménagement et de Gestion (2017). Plan d'Aménagement et de Gestion de la RNI (2017 - 2026). Elaboré par l'ICCN - RNI et ses partenaires en collaboration avec Frédéric AIRAUD - Consultant spécialisé en Environnement ; Kinshasa, 99 p.

38. Pinton, F. \& Grenand, P. (2007). « Savoirs traditionnels, populations locales et ressources globalisées », Les marchés de la biodiversité, IRD Éditions $165-263 p$

39. Plumptre, A., Amsini, F., Kujirakwinja, D., Hart, J., Nyembo, B., Vyahavwa, C., Bujo, F., Masanga, A., Matunguru, J., Mwinyihali, R. \& Tshombe, R. (2009). Itombwe Massif conservation Project; Delimitation and zoning of the Itombwe natural Reserve for protection for protection of great apes. Unpublished Report to US Fish and Wildlife Service. Project $98210-7-$ G293, Web site : http://www.wcs.org

40. Prigogine, A. (1985). Conservation of the avifauna of the forests of the Albertine Rift. In: Conservation of Tropical Forest birds. International council for Bird Preservation Technical Publication No. 4.pp 277-295 Birdlife International, Cambridge.

41. Roué, M. (2012). " Histoire et épistémologie des savoirs locaux et autochtones », Revue d'ethnoécologie[En ligne], $1 \mid 2012$, mis en ligne le 02 décembre 2012, Consulté le 30 septembre 2016. URL : http:// ethnoecologie.revues.org/813

http://dx.doi.org/10.4000/ethnoecologie.813.

42. Roussel, B. (2003). La Convention sur la diversité biologique : les savoirs locaux au coeur des débats internationaux. Les synthèses de l'Iddri, n² 2 , octobre ;

http://www.iddri.org/Publications/Collections/Syntheses/sy_0302_ro ussel.pdf

43. Roussel, B. (2005). « Savoirs locaux et conservation de la biodiversité : renforcer la représentation des communautés. », Mouvements, vol. 4, 
no 41, pp. 82-88 URL : www.cairn.info/revue-mouvements-2005-4page-82.htm.

44. Sanou, B.D. (2010). Aménagement des forêts de Dinderesso et du Kou et autoreprésentation des communautés villageoises riveraines. Proposition d'une méthode d'actualisation des coutumes pour une gestion durable des ressources naturelles. Bobo-Dioulasso, Burkina Faso.

45. Sene, C., Dioh, P., \& Mormont, M. (2013). « Pratiques traditionnelles et gestion durable des ressources naturelles : Etude de cas des sites naturels sacrés côtiers et marins à Jaol-Fadiouth », ASRDLF - Appel à communication - Feuille de style, 18.

46. Tardío, J. \& Pardo-de-Santayana, M. (2008). Cultural Importance Indices : A Comparative Analysis Based on the Useful Wild Plants of Southern Cantabria (Northern Spain). Economic Botany, 62(1), 24-39. https://doi.org/10.1007/s12231-007-9004-5

47. Touré, E.A. (2010). « Réflexion épistémologique sur l'usage des focus groups : fondements scientifiques et problèmes de scientificité », Dans Guillemette F., Luckerhoff J. et Baribeau C.,Entretiens de groupe : concepts, usages et ancrages, Recherches qualitatives, 29 (1), 5-27.

48. Wilson, J.R. \& Catsis, M.C. (1990). A Preliminary survey of the forests of the 'Itombwe' Mountains and the Kahuzi-Biega National Park Extension, East Zaire, July-Sept 1989. Unpublished report to WWF:FFPS:IZCN, Project 3902, Kinshasa

49. Yombatina, B. (2000). Droit de l'environnement à l'épreuve des représentations culturelles africaines : la nécessité d'une approche dialectique et plus responsable, Mémoire de Master, Université SaintLouis, Bruxelles 